\title{
STABILITY INVESTIGATION OF QUADRATIC SYSTEMS WITH DELAY
}

\author{
VLADIMIR DAVYDOV and DENYS KHUSAINOV \\ Kiev University \\ Department of Complex Systems Modelling \\ 64 Vladimirskaya Street \\ Kiev, Ukraine 252033 \\ E-mail: denis@dh.cyb.univ.kiev.ua
}

(Received January, 1997; Revised March, 1999)

\begin{abstract}
Systems of differential equations with quadratic right-hand sides with delay are considered in the paper. Compact matrix notation form is proposed for the systems of such type. Stability investigations are performed by Lyapunov's second method with functions of quadratic form. Stability conditions of quadratic systems with delay, uniformly by argument deviation, and with delay depending on the system's parameters are derived. A guaranteed radius of the ball of asymptotic stability region for zero solution is obtained.
\end{abstract}

Key words: Quadratic Differential System, Lyapunov Function, Asymptotic Stability, Delay.

AMS subject classifications: $34 \mathrm{~K} 20$.

\section{Introduction}

In this paper, sufficient conditions for asymptotic stability of a differential-delay equations systems with quadratic right-hand sides are given. Compact matrix form from presentation of the quadratic differential-delay system is proposed. Lyapunov's second method is taken as the main method of our investigations. There are two approaches to the application of Lyapunov's second method for differential equations. The first one is the Lyapunov function (or direct) method. It involves a search for functions from the class of functions with a finite number of variables satisfying Lyapunov's theorems conditions. The second approach is Lyapunov's functional method stressing the point of view of functional analysis. This method is linked with a search for functionals defined on integral curve segments. Essential difficulties in applications of Lyapunov's direct method to differential equations with deviating arguments were formulated for the first time by L.E. El'sgol'ts in [5]. The main difficulties lay in a sign estimate of Lyapunov function derivative, which are functions of $2 n$-variables, if $n$th order delay-differential systems are considered. Important steps to overcome these difficulties were made by B.S. Razumikhin [6, 7]. The following 
sufficient condition for stability was obtained.

If there exists a positive definite function $V(t, x), x \in E_{n}$, the total derivative of the system is a negative definite functional on curves respecting solutions and satisfying the Razumikhin's condition $V(\delta, x(\delta)) \leq V(t, x(t)), \delta<t, t \geq t_{0}$; if the set of the curves is not empty, then the system is stable. Note that this condition is not an addition for reducing the class of stability tasks. The condition allows to get an estimate of Lyapunov function derivatives in a more simple way. So some efficient conditions of stability and asymptotic stability have been given in [6] by using Razumikhin's condition.

The rich experience with Lyapunov functions construction for ordinary differential equations explains the wide application of Lyapunov's direct method for systems with deviating arguments.

\section{Main Results}

A system of differential-delay equations with quadratic right-hand sides is considered:

$$
\begin{aligned}
& \dot{x}(t)=A_{1} x(t)+A_{2} x(t-\tau)+X(t) B_{1} x(t) \\
& +X(t-\tau) B_{2} x(t)+X(t-\tau) B_{3} x(t-\tau),
\end{aligned}
$$

where $\tau>0$ is a constant delay; $A_{1}, A_{2}$ are square $n \times n$ matrices with constant coefficients; $X(t)$ and $B_{j}^{T}, j=\overline{1,3}$ are rectangular $n \times n^{2}$ matrices of the following block forms:

$$
X(t)=\left\{X_{1}(t), X_{2}(t), \ldots, X_{n}(t)\right\}, B_{j}^{T}=\left\{B_{1 j}, B_{2 j}, \ldots, B_{n j}\right\}
$$

where $X_{i}(t)$ is a square $n \times n$ matrix containing the vector $x^{T}(t)=\left(x_{1}(t), x_{2}(t)\right.$, $\left.\ldots, x_{n}(t)\right)$ at the $i$ th row of the matrix and the other elements being zero, $B_{i j}$ are square-symmetric matrices characterizing the quadratic component of the $i$ th-line of the system (1). That is,

$$
X_{i}(t)=\left(\begin{array}{cccc}
0 & 0 & \ldots & 0 \\
0 & 0 & \ldots & 0 \\
\ldots & \ldots & \ldots & \ldots \\
x_{1}(t) & x_{2}(t) & \ldots & x_{n}(t) \\
0 & 0 & \ldots & 0 \\
\ldots & \ldots & \ldots & \ldots \\
0 & 0 & \ldots & 0
\end{array}\right), B_{i j}=\left(\begin{array}{cccc}
b_{11}^{i j} & b_{12}^{i j} & \ldots & b_{1 j} \\
b_{12}^{i j} & b_{22}^{i j} & \ldots & b_{2 n}^{i j} \\
\ldots & \ldots & \ldots & \ldots \\
b_{1 n}^{i j} & b_{2 n}^{i j} & \ldots & b_{n n}^{i j}
\end{array}\right)
$$

Such notation of a quadratic differential system in matrix form (1) was proposed in the articles $[8,9]$. This form is convenient for investigation of quadratic systems of a general form.

The conclusion about stability of the zero solution of (1) is performed on the basis of comparison of the differential system with delay (1) with some model system. As a model system we take a linear system without delay 


$$
\dot{x}(t)=A x(t), \quad A=A_{1}+\beta A_{2},
$$

where $\beta, 0 \leq \beta \leq 1$ is a certain numerical parameter selected with respect to the condition of maximum stability of the system (2). If the model system (2) is asymptotically stable then this property is retained by the zero solution of the system (1) in some sufficiently small neighborhood of the equilibrium position. Let the matrix $A$ be asymptotically stable, i.e., all eigenvalues of the matrix are on the left of the imaginary axis. Then for an arbitrary positive definite symmetric matrix $C$ the equation

$$
A^{T} H+H A=-C
$$

has as unique solution a positive definite matrix $H$ [2]. Stability investigation of the solution $x(t) \equiv 0$ is performed by Lyapunov's second (direct) method. Take the quadratic form $v(x)=x^{T} H x$ as Lyapunov function, where the positive definite matrix $H$ is a solution of (3). For the function $v(x)$, the following two-sided inequality is true:

$$
\lambda_{\min }(H)|x|^{2} \leq v(x) \leq \lambda_{\max }(H)|x|^{2} .
$$

Here and further on $\lambda_{\min }(\cdot)$ respectively $\lambda_{\max }(\cdot)$ are the largest respectively the smallest eigenvalues of positive definite matrices.

Let us denote a level surface of the Lyapunov function $v(x)$ by $\partial v^{\alpha}$, and a region bounded by this surface as $v^{\alpha}$, that is,

$$
\partial v^{\alpha}=\{(x, t): v(x, t)=\alpha\}, v^{\alpha}=\{(x, t): v(x, t)<\alpha\} .
$$

The following vector norms are used:

$$
|x(t)|=\left\{\sum_{i=1}^{n} x_{i}^{2}(t)\right\}^{1 / 2}, \quad\|x(t)\|_{\tau=\tau \leq s \leq 0}\{|x(t+s)|\} .
$$

The spectral norm is taken as a matrix norm: $|A|=\left\{\lambda_{\max }\left(A^{T} A\right)\right\}^{1 / 2}$.

Now we introduce some important definitions.

Definition 1: The asymptotic stability region $\Omega$ of the zero solution of the system with delay is the set of initial functions $x(t),-\tau \leq t \leq 0$, such that

$$
\Omega=\left\{x(t),-\tau \leq t \leq 0, \lim _{t \rightarrow \infty}|x(t)|=0\right\} .
$$

Definition 2: The set of initial function values $x(t) \in \Omega$ located inside the ball

$$
U_{R}=\{x(t): x(t) \in \Omega,|x(t)|<R\}
$$

is said to be the ball $U_{R}$ of radius $R$ of the asymptotic stability region.

Theorem 1: Let there exist a parameter $\beta, 0 \leq \beta \leq 1$ and matrix $H$ satisfying the equation (3), such that the inequality

$$
\lambda_{\min }(C)-2\left|H A_{2}\right|(\beta+\varphi(H))>0
$$

holds where $\varphi(H)=\sqrt{\lambda_{\max }(H) / \lambda_{\min }(H)}$. Then the solution $x(t) \equiv 0$ of the system 
with delay (1) is asymptotically stable for arbitrary $\tau>0$. The ball $U_{R}$ of the asymptotic stability region has the radius

$$
R=\frac{\lambda_{\min }(C)-2\left|H A_{2}\right|(\beta+\varphi(H))}{2 \lambda_{\max }(H) \sum_{i=1}^{3}\left|B_{i}\right| \varphi^{i}(H)}
$$

Moreover, an arbitrary solution $x(t)$ does not leave $\varepsilon$-neighborhoods of the origin, that is, $|x(t)|<\varepsilon, t>0$, if $\|x(0)\|_{\tau}<\delta(\varepsilon)$, where

$$
\delta(\varepsilon)=\min \{R, \varepsilon / \varphi(H)\}
$$

Proof: Let $\varepsilon>0$ be an arbitrary value and $x(t)$ a solution of the system (1) such that the condition $\|x(0)\|_{\tau}<\delta(\varepsilon)$ is satisfied, where the function $\delta(\varepsilon)$ is chosen according to (7). Then the solution $x(t),-\tau \leq t \leq 0$, is located inside the region $v^{\alpha}$, where $\alpha=\lambda_{\max }(H) \delta^{2}(\varepsilon)$. We show that $x(t) \in v^{\alpha}$ for all $t \geq 0$. Otherwise, there is some minimum value of $S>0$ where $x(t)$ reaches the first time the boundary $\partial v^{\alpha}$, $x(S) \in \partial v^{\alpha}$. Consider the total derivative of the Lyapunov function $v(x)$ along the solutions of the system (1):

$$
\begin{aligned}
& \dot{v}(x(t))=-x^{T}(t) C x(t)+2 x^{T}(t) H\left\{A_{2}[x(t-\tau)-\beta x(t)]\right. \\
& \left.+X(t) B_{1} x(t)+X(t-\tau) B_{2} x(t)+X(t-\tau) B_{3} x(t-\tau)\right\}
\end{aligned}
$$

Exploiting the special form of the matrices $X(t), X(t-\tau)$ and the above-mentioned spectral norm for matrices, we obtain

$$
|X(t)|=|x(t)|,|X(t-\tau)|=|x(t-\tau)|
$$

Therefore, the estimate for the total derivative of the Lyapunov function is fulfilled:

$$
\begin{gathered}
\dot{v}(x(t))<-\lambda_{\min }(C)|x(t)|^{2}+2\left\{\left|H A_{2}\right|[|x(t-\tau)|+\beta|x(t)|]\right. \\
\left.+|H|\left[\left|B_{1}\right||x(t)|^{2}+\left|B_{2}\right||x(t)||x(t-\tau)|+\left|B_{3}\right||x(t-\tau)|^{2}\right]\right\}|x(t)| .
\end{gathered}
$$

According to our assumption, $x(t) \in v^{\alpha}$ for any $t<S$ and $S: x(S) \in \partial v^{\alpha}$, yielding

$$
\lambda_{\min }(H)|x(t)|^{2} \leq v(x(t))<v(x(S)) \leq \lambda_{\max }(H)|x(S)|^{2} .
$$

From this, it follows that

$$
|x(t)|<\varphi(H)|x(S)|, \quad \varphi(H)=\sqrt{\lambda_{\max }(H) / \lambda_{\min }(H)} .
$$

Therefore, for the total derivative of the Lyapunov function at $t=S$ holds the inequality

$$
\begin{gathered}
\dot{v}(x(S))<-\left\{\lambda_{\min }(C)-2\left|H A_{2}\right|(\beta+\varphi(H))\right\}|x(S)|^{2} \\
|H|\left[\left|B_{1}\right|+\left|B_{2}\right| \varphi(H)+\left|B_{3}\right| \varphi^{2}(H)\right]|x(t)|^{3}
\end{gathered}
$$


Furthermore, if

$$
|x(S)|<\frac{\lambda_{\min }(C)-2\left|H A_{2}\right|(\beta+\varphi(H))}{2 \lambda_{\max }(H) \sum_{i=1}^{3}\left|B_{i}\right| \varphi^{i}(H)},
$$

then the total derivative is negative definite. Thus, according to Theorem 1 , the ball $U_{R}$ of the asymptotic stability region is the interior ball with radius $R$ defined in (6). Thus, the proof is completed.

For defining the largest radius $R$ of the ball a similar remark holds true.

Stability conditions uniformly in the delay $\tau>0$ proposed in Theorem 1 have an excessively sufficient character. They impose strong restrictions on the system (1). If the model system (2) is asymptotically stable, then the zero solution of the system (1) will be asymptotically stable without satisfying the conditions of Theorem 1. However this is true only for sufficiently small delays $\tau<\tau_{0}$, where $\tau_{0}$ is the admissible maximum value of delay depending on the quadratic system parameters and on the choice of the matrix $H$.

For deduction of these conditions it is necessary to estimate the value of maximum deviation of solutions from the equilibrium position in one step. In contrary to linear systems, quadratic ones have the property of nonextensibility, i.e. in finite time their solutions may go to infinity. This property holds even for simple scalar equations. Therefore, we find conditions for the system parameters, delay $\tau$ and the value of initial perturbation $\delta$, under which it is possible to estimate the maximum deviation of the solution $x(t)$ of $(1)$ on the interval $0 \leq t \leq \tau$. Set:

$$
P=\left[1+\left(\left|A_{2}\right|+\left|B_{3}\right| \delta\right) \tau\right] \delta, \quad L=\left|A_{1}+\right| B_{2}|\tau, Q=| B_{1} \mid
$$

Lemma 1: Let $\delta>0$ and $\tau>0$, such that the inequality

$$
\frac{L}{P\left|B_{1}\right|}+1>\exp \{L \tau\}
$$

holds. Then for arbitrary solutions $x(t)$ of $(1)$ satisfying the condition $\|x(0)\|_{\tau}<\delta$, for $0 \leq t \leq \tau$ the following relation is fulfilled:

$$
|x(t)| \leq \frac{P \operatorname{Lexp}\{L t\}}{L+P\left|B_{1}\right|(1-\exp \{L t\})}
$$

Proof: Rewrite the system (1) in the integral form

$$
\begin{aligned}
x(t) & =x(0)+\int_{0}^{t}\left[A_{1} x(s)+A_{2} x(s-\tau)+X(s) B_{1} x(s)\right. \\
& \left.+X(s-\tau) B_{2} x(s)+X(s-\tau) B_{3} x(s-\tau)\right] d s .
\end{aligned}
$$

For $0 \leq t \leq s$ the following estimate holds:

$$
\begin{gathered}
|x(t)| \leq\left[1+\left(\left|A_{2}\right|+\left|B_{3}\right| \delta\right) \tau\right] \delta \\
+\int_{0}^{t}\left[\left(\left|A_{1}\right|+\left|B_{2}\right| \delta\right)|x(s)|+\left|B_{1}\right||x(s)|^{2}\right] d s
\end{gathered}
$$


From Bihari's Lemma [3] it follows that if the inequalities

$$
u(t) \leq P+\int_{0}^{t} f(u(\xi)) d \xi, \quad u(t) \geq 0,
$$

hold, then the relation $u(t) \leq \Psi^{-1}(t)$ is true, where $\Psi^{-1}(t)$ is an inverse function to

$$
\Psi(u)=\int_{P}^{u} \frac{d z}{F(z)}
$$

Using the settings introduced above, we obtain

$$
F(z)=z(Q z+L), \quad \Psi(u)=\frac{1}{L} \ln \left[\frac{u(Q P+L)}{P(Q u+L)}\right]
$$

And consequently,

$$
\Psi^{-1}(t)=\frac{P L \exp \{L t\}}{L+P Q(1-\exp \{L t\})}
$$

For the solution $x(t)$ of (1) per time interval $0 \leq t \leq \tau$ not to go to infinity it is sufficient that the denominator in (9) is positive, that is, the condition (8) should be satisfied.

Lemma 2: Let $x(S) \in \partial v^{\alpha}$ and $x(s) \in v^{\alpha}$ be fulfilled for $-\tau \leq s<S, S>\tau$. Then the following inequality holds:

$$
\begin{aligned}
|x(S)-x(S-\tau)| & \\
& \leq\left[\left|A_{1}\right|+\left|A_{2}\right|+\sum_{i=1}^{3}\left|B_{i}\right| \varphi(H) \mid x(S)\right)|\varphi(H)| x(S) \mid \tau .
\end{aligned}
$$

Proof: Rewrite the quadratic system (1) in the form

$$
\begin{gathered}
x(t)=x(t-\tau)+\int_{t-\tau}^{t}\left[A_{1} x(s)+A_{2} x(s-\tau)+X(s) B_{1} x(s)+X(s-\tau) B_{2} x(s)\right. \\
\left.\quad+X(s-\tau) B_{3} x(S-\tau)\right] d s .
\end{gathered}
$$

If $t=S$, then we derive

$$
\begin{aligned}
& |x(S)-x(S-\tau)| \leq \int_{S-\tau}^{S}\left[\left|A_{1}\right||x(s)|+\left|A_{2}\right||x(s-\tau)|+\left|B_{1}\right||x(s)|^{2}\right. \\
& \left.\quad+\left|B_{2}\right||x(s)||x(s-\tau)|+\left|B_{3}\right||x(s-\tau)|^{2}\right] d s .
\end{aligned}
$$

Since $x(s) \in v^{\alpha}$, the relation (10) holds true and the proof is completed.

We use these lemmata for obtaining stability conditions of the solution $x(t) \equiv 0$ of 
the system with quadratic right-hand sides and a delay depending on the system parameters. Let $\beta=1$, then the matrix of the model system is of the form $A=A_{1}+A_{2}$.

For rendering the following results more compact, introduce the function

$$
N(\varepsilon, \tau)=\frac{\left(\left|A_{1}\right|+\left|B_{2}\right| \tau\right) \varepsilon / \varphi(H)}{\left(\left|A_{1}\right|+\left|B_{2}\right| \tau+\left|B_{1}\right| \varepsilon / \varphi(H)\right) \exp \left\{\left(\left|A_{1}\right|+\left|B_{2}\right| \tau\right) \tau\right\}-\left|B_{1}\right| \varepsilon / \varphi(H)}
$$

Theorem 2: Let $A$ be an asymptotically stable matrix. Then for $\tau<\tau_{0}$, where

$$
\tau_{0}=\frac{\lambda_{\min }(C)}{2\left|H A_{2}\right|\left(\left|A_{1}\right|+\left|A_{2}\right|\right) \varphi(H)}
$$

the system (1) has an asymptotically stable zero solution. The asymptotic stability region contains the ball with radius $R=\delta(\bar{R}, \tau)$, where

$$
\bar{R}=\frac{\lambda_{\min }(C)\left(1-\tau / \tau_{0}\right)}{2 \sum_{i=1}^{3}\left[\left|H A_{2}\right|\left|B_{i}\right| \varphi^{3}(H) \tau+\lambda_{\max }(H)\left|B_{i}\right| \varphi^{i}(H)\right]} .
$$

Moreover, for arbitrary solutions $x(t), t>0$, the relation $|x(t)|<\varepsilon$ holds, where $0<\varepsilon<\bar{R} \varphi(H)$, only if $\|x(0)\|_{\tau}<\delta(\varepsilon, \tau)$, where

$$
\delta(\varepsilon, \tau)=\left\{\begin{array}{cl}
N(\varepsilon, \tau)\left[1+\left|A_{2}\right| \tau\right]^{-1}, & \text { if }\left|B_{3}\right|=0 \\
2 N(\varepsilon, \tau)\left[\sqrt{\left(1+\left|A_{2}\right| \tau\right)^{2}+4 N(\varepsilon, \tau)\left|B_{3}\right| \tau}\right. & \text { if }\left|B_{3}\right| \neq 0
\end{array}\right.
$$

Proof: For arbitrary $\varepsilon, 0<\varepsilon<\bar{R} \varphi(H)$ set $\alpha=\lambda_{\min }(H) \varepsilon^{2}$. Then the level surface $v(x)=\alpha$ of the Lyapunov function $v(x)=x^{T} H x$ is contained inside the $\varepsilon$-neighborhood of the origin. Further, chose $\delta(\varepsilon, \tau)$ according to (13). Then the solution $x(t)$ satisfying the condition $\|x(0)\|_{\tau}<\delta(\varepsilon, \tau)$ in the interval $0 \leq t \leq \tau$ does not leave the $\varepsilon / \varphi(H)$-neighborhood of the origin and is contained in the region $v^{\alpha}$.

We show that $x(t) \in v^{\alpha}$ for all $t>\tau$. Otherwise, there would exist $S>\tau: x(S) \in$ $\partial v^{\alpha}$, and $x(t) \in v^{\alpha}$ for $-\tau \leq t<S$. Calculate the total derivative of the Lyapunov function $v(x)=x^{T} H x$ along the solutions $x(t)$ of $(1)$ at $t=S$ :

$$
\begin{gathered}
\dot{v}(x(S))=-x^{T}(S) C x(S)+2 x^{T}(S) H A_{2}[x(S-\tau)-x(S)] \\
+2 x^{T}(S) H\left[X(S) B_{1} x(S)+X(S-\tau) B_{2} x(S)+X(S-\tau) B_{3} x(S-\tau)\right] .
\end{gathered}
$$

Using the inequality (10), the assumption $x(S) \in \partial v^{\alpha}$ and the relation $|x(t)|<$ $\varphi(H)|x(S)|$ for all $t<S$, we obtain

$$
\begin{aligned}
& \dot{v}(x(S)) \leq-\left[\lambda_{\min }(C)-2\left|H A_{2}\right|\left(\left|A_{1}\right|+\left|A_{2}\right|\right) \varphi(H) \tau\right]|x(S)|^{2} \\
& +2\left\{\left|H A_{2}\right| \sum_{i=1}^{3}\left|B_{i}\right| \varphi(H) \tau+|H| \sum_{i=1}^{3}\left|B_{i}\right| \varphi^{i-1}(H)\right\}|x(S)|^{3} .
\end{aligned}
$$


Let $\tau<\tau_{0}$, with $\tau_{0}$ defined as in (11). Then we have the estimate:

$$
\begin{gathered}
\dot{v}(x(S)) \leq-\lambda_{\min }(C)\left(1-\tau / \tau_{0}\right)|x(S)|^{2} \\
+2\left\{\left|H A_{2}\right| \sum_{i=1}^{3}\left|B_{i}\right| \varphi(H) \tau+|H| \sum_{i=1}^{3}\left|B_{i}\right| \varphi^{i-1}(H)\right\}|x(S)|^{3} .
\end{gathered}
$$

From this it follows that if the inequality

$$
|x(S)| \leq \frac{\lambda_{\min }(C)\left(1-\tau / \tau_{0}\right)}{2 \sum_{i=1}^{3}\left|B_{i}\right|\left[\left|H A_{2}\right| \varphi(H) \tau+|H| \varphi^{i-1}(H)\right]}
$$

holds, then the total derivative of $v(x)$ is a negative definite function. Taking into consideration the inequalities for quadratic forms (4) we obtain the expression (12) for the radius $R$ of the corresponding ball in the asymptotic stability region. Thus, the theorem is proved.

\section{References}

[1] Hale, J., Theory of Functional Differential Equations, Springer Verlag, New York-Heidelberg-Berlin 1977.

[2] Barbashin, E.A., Lyapunov Functions, Nauka, Moscow 1970. (Russian)

[3] Bihari, I., A generalization of a lemma of Bellman and its applications to uniqueness problem of differential equation, Acta Math. Acad. Sci. Hung. 7 (1954), 81-94.

[4] El'sgol'ts, L.E., Introduction to the Theory of Differential Equations with Deviating Arguments, Holden-Day, San Francisco 1966.

[5] El'sgol'ts, L.E., Stability of solution of differential-equations, Uspehi Mat. Nauk. 9:4 (1954), 95-112. (Russian)

[6] Razumikhin, B.S., About stability of systems with delay, Prikladnaya Mat. $i$ Meh. 20:4 (1956), 500-512. (Russian)

[7] Razumikhin, B.S., Method of stability investigation with post-action, $D A N$ USSR 167:6 (1966), 1234-1237.

[8] Khusainov, D. Ya. and Davydov, V.F., Stability of delayed systems of quadratic form, DAN Ukrainy 7 (1994), 11-13.

[9] Davydov, V.F., Marjozing estimates of solutions of quadratric differential systems with delay, Ukrain. Math. Zurn. 47:4 (1995), 60-68. 


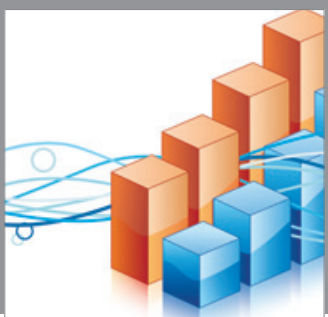

Advances in

Operations Research

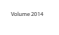

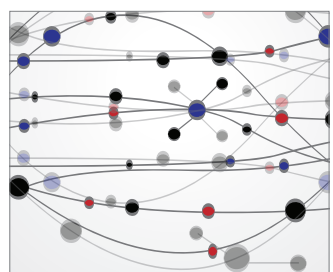

\section{The Scientific} World Journal
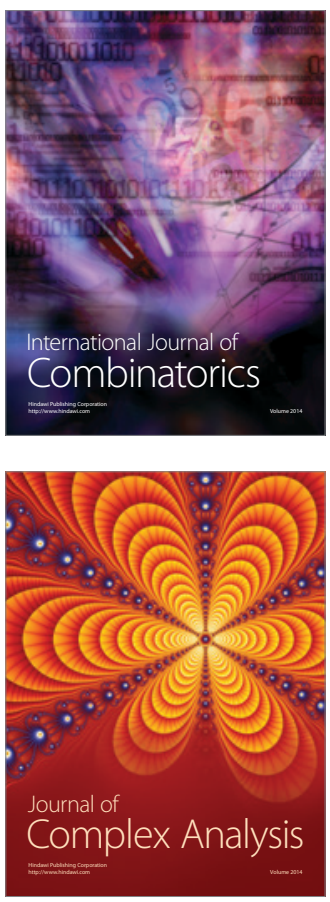

International Journal of

Mathematics and

Mathematical

Sciences
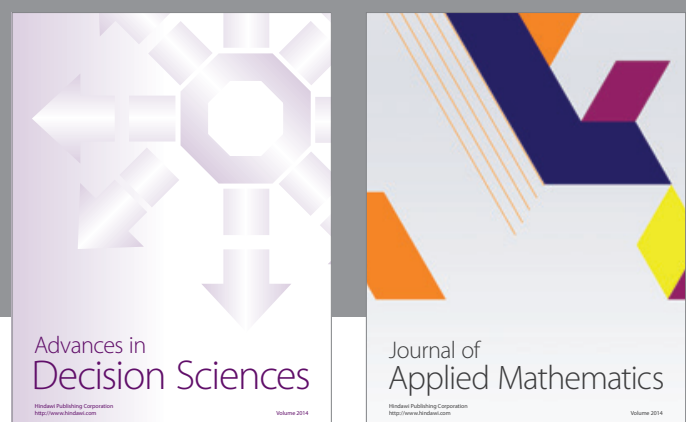

Journal of

Applied Mathematics
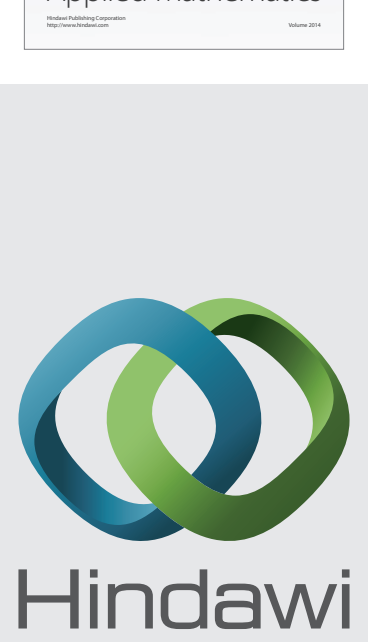

Submit your manuscripts at http://www.hindawi.com
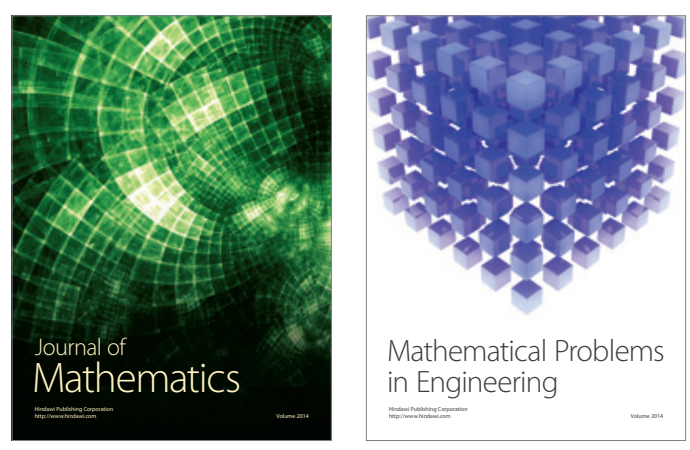

Mathematical Problems in Engineering
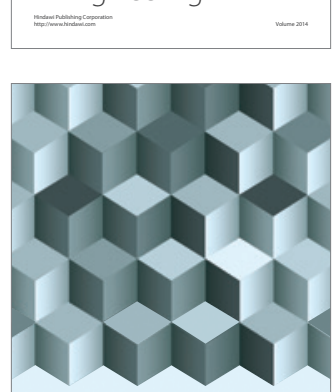

Journal of

Function Spaces
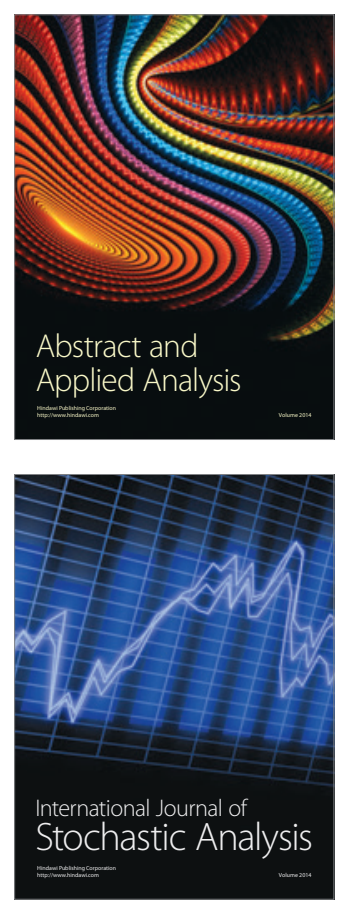

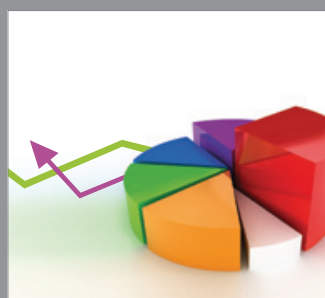

ournal of

Probability and Statistics

Promensencen
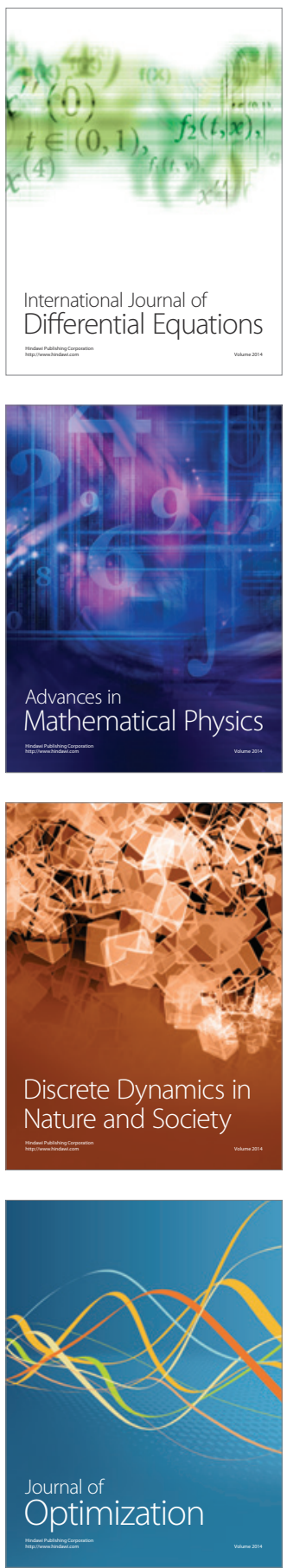